\title{
Stéréotypes prescriptifs et avantages des groupes dominants
}

\author{
Nathalie Delacollette ${ }^{1 \star}$, Benoit Dardenne ${ }^{1}$ et Muriel Dumont ${ }^{1,2}$ \\ ${ }^{1}$ Université de Liège, Belgique \\ ${ }^{2}$ Université de Louvain-la-Neuve, Belgique
}

\section{RÉSUMÉ}

La composante prescriptive des stéréotypes est définie comme un ensemble de croyances à propos des caractéristiques auxquelles les membres d'un groupe doivent se conformer. Elle a été principalement étudiée dans le cadre des stéréotypes sexuels. Nous pensons que la fonction principale de cette composante prescriptive est de permettre aux membres des groupes dominants de maintenir les membres des groupes subordonnés dans une position qui leur est avantageuse. Nous illustrons notre propos en présentant différents articles théoriques et empiriques qui montrent que le statut relatif de différents groupes sociaux détermine en grande partie les caractéristiques prescrites aux membres de ces groupes, que les subordonnés qui ne se conforment pas aux prescriptions dont ils font l'objet sont sanctionnés, et que les dominants prescrivent aux subordonnés des caractéristiques qu'ils jugent bénéfiques pour leur propre groupe.

\section{Prescriptive stereotypes and dominant groups' benefits}

\begin{abstract}
The prescriptive component of stereotypes is defined as a set of beliefs about the characteristics group members should possess. It has mainly been studied regarding gender stereotypes. We believe that the main function of this prescriptive component is to allow dominant group members to maintain subordinate group members in an advantageous position for themselves. To illustrate our proposal, we present several theoretical and empirical papers, showing that the relative status of social groups is a determinant of which characteristics are prescribed to the members of these groups; that subordinates who fail to conform to the prescription are sanctioned; and that dominants prescribe to subordinates characteristics they see as beneficial to their own group.
\end{abstract}

*Correspondance : Psychologie Sociale, Département des Sciences Cognitives, Université de Liège, 5, Boulevard du Rectorat (B32), 4000 Liège, Belgique. E-mail : ndelacollette@ulg.ac.be 
L'étude du contenu des stéréotypes n'est pas neuve. Bien que de nombreuses définitions existent, on pourrait définir ceux-ci comme « un ensemble de croyances partagées à propos des caractéristiques personnelles, généralement des traits de personnalité, mais aussi des comportements, propres à un groupe de personnes » (Leyens, Yzerbyt \& Schadron, 1994). Jusque dans les années 1950, les stéréotypes étaient considérés comme des images produites culturellement et étroitement liées aux préjugés et à la discrimination (Schneider, 2004). Les études initiales qui portent sur ces images culturelles vont s'attacher à établir leur contenu. Les premières études empiriques à propos des stéréotypes datent ainsi des années 1930 et leur objectif principal est de décrire l'attribution de traits à certains groupes (Schneider, 2004). Par exemple, dans leur étude aujourd'hui célèbre, Katz et Braly (1933) ont demandé à des étudiants d'indiquer dans quelle mesure différents groupes ethniques et nationaux possédaient une série de traits. Les premières recherches sur les stéréotypes avaient donc pour objectif la pure et simple description de leur contenu. Cependant, aucune attention n'était portée aux régularités susceptibles d'apparaitre au sein de ce contenu, ni aux dimensions qui pourraient structurer celui-ci, encore moins aux processus sous-tendant la formation des stéréotypes. Ces questions ne seront abordées que bien plus tard. À partir des années 1950, les stéréotypes sont principalement considérés comme des manifestations de préjugés et la recherche se centre sur la personnalité des individus qui ont le plus tendance à utiliser les stéréotypes (par exemple, la «personnalité autoritaire » décrite par Adorno, Frenkel-Brunswik, Levinson \& Sanford, 1950). Jusqu'aux années 1970, la recherche sur les stéréotypes reste donc très descriptive et se centre principalement sur les stéréotypes ethniques ainsi que sur les individus qui y adhèrent. À partir des années 1970 se développe la perspective de la cognition sociale, qui donne un nouveau souffle à la recherche sur les stéréotypes, en focalisant son attention cette fois sur les processus cognitifs de stéréotypisation plutôt que sur le contenu des stéréotypes ou sur les personnalités particulièrement susceptibles de les utiliser (voir par exemple Hamilton \& Stroessner, 1994 ; Leyens, Yzerbyt \& Schadron, 1994 ; Spears, Oakes, Ellemers \& Haslam, 1997 ; Yzerbyt \& Schadron, 1994 ; Wyer, 1998). Les stéréotypes sont alors considérés comme des structures de connaissances abstraites qui peuvent être utilisées par tout individu et la recherche se focalise sur l'étude des processus cognitifs liés à la formation et à l'utilisation des stéréotypes. Bien que l'on voie se développer un intérêt pour les problématiques de la discrimination raciale ou sexuelle (Schneider, 2004), le contenu des stéréotypes passe au second plan, au profit de l'étude des processus et des mécanismes de stéréotypisation. Un certain intérêt pour l'étude du contenu des stéréotypes 
est réapparu récemment. Toutefois, les chercheurs portent à présent leur attention sur les régularités systématiques qui peuvent apparaître au niveau de ce contenu (Judd, James-Hawkins, Yzerbyt \& Kashima, 2005) et sur les dimensions qui le sous-tendent (Alexander, Brewer \& Herrmann, 1999 ; Fiske, Cuddy, Glick \& Xu, 2002 ; Phalet \& Poppe, 1997). Il apparaît donc que l'intérêt pour la structure du contenu des stéréotypes est relativement récent.

Ce n'est que très récemment que s'est développé un intérêt pour la distinction entre les composantes prescriptive et descriptive des stéréotypes. La composante descriptive renvoie, comme son nom l'indique, aux croyances à propos des caractéristiques qui décrivent les membres d'un groupe. La composante prescriptive, quant à elle, consiste en un ensemble de croyances à propos des caractéristiques auxquelles les membres d'un groupe doivent se conformer (Burgess \& Borgida, 1999). Dans le présent article, nous nous focaliserons sur la composante prescriptive. Nous pensons qu'elle reflète ce que les groupes dominants attendent des individus, et spécialement des membres des groupes subordonnés. Plus précisément, alors que la composante descriptive n'a d'autre fonction que de décrire, la fonction de la composante prescriptive des stéréotypes serait de maintenir stable l'ordre social établi et de permettre aux dominants de maintenir les subordonnés dans une position qui leur est avantageuse. Ces derniers, s'ils ne respectent pas les prescriptions dont ils font l'objet, sont alors susceptibles d'être sanctionnés.

L'objectif du présent article est donc de démontrer, en passant en revue différents articles théoriques et empiriques, que les membres des groupes dominants prescrivent aux membres des groupes subordonnés des caractéristiques qui leur sont particulièrement bénéfiques. Cette idée, bien que développée de manière théorique dans la littérature, a été peu étudiée de manière empirique et, de plus, a été abordée de manière très morcelée. Nous nous attacherons donc à rassembler les données empiriques susceptibles de nous éclairer sur la fonction de la composante prescriptive des stéréotypes et de mettre en lumière le lien entre l'avantage des dominants à ce que les subordonnés possèdent certains traits et la prescription de ces traits. Les stéréotypes de genre ayant été les plus abordés, c'est donc sur ceux-ci que portera particulièrement notre réflexion.

Le contenu des stéréotypes, et par conséquent leur composante prescriptive également, est généralement abordé et mesuré par rapport à deux dimensions importantes : la compétence et la sociabilité. Les groupes sociaux sont souvent évalués de manière ambivalente sur ces deux dimensions (Glick \& Fiske, 1999 ; 2001 ; Fiske, Xu, Cuddy \& Glick, 1999). Nous commencerons donc par les présenter brièvement. 
Nous aborderons ensuite la notion d'ambivalence au travers de la théorie des stéréotypes ambivalents (Glick \& Fiske, 1999 ; 2001). Nous nous pencherons également sur ce que cette théorie nous apprend en termes de relations entre groupes dominants et subordonnés, puis nous verrons de quelle manière ces relations intergroupes sont susceptibles d'influencer la composante prescriptive des stéréotypes, et ce particulièrement en ce qui concerne les stéréotypes de genre. Nous verrons ainsi que le statut relatif des groupes sociaux (dominant ou subordonné) et le type de relation qu'ils entretiennent (coopérative ou compétitive) détermine en grande partie le contenu de la composante prescriptive des stéréotypes. Nous aborderons ensuite le lien entre violation des prescriptions stéréotypiques et sanction. Enfin, nous verrons en quoi la composante prescriptive des stéréotypes peut être vue comme un moyen de maintenir l'ordre social établi et les avantages du groupe dominant.

\section{LES DIMENSIONS DE SOCIABILITÉ ET COMPÉTENCE}

On sait aujourd'hui que la plupart des jugements sociaux sont sous-tendus par deux dimensions : la compétence d'une part et la sociabilité d'autre part (Judd et al., 2005). La dimension de sociabilité renvoie à une orientation vers les autres, elle comprend des traits tels que la douceur, l'empathie, la sensibilité ou encore la politesse. La dimension de compétence est orientée vers la réalisation d'une tâche, l'atteinte d'un objectif. Elle renvoie donc à des traits davantage tournés vers l'action tels que la compétitivité, l'assertivité, l'ambition ou les capacités de leadership (voir par exemple Glick \& Fiske, 1999 ; 2001). Ces deux dimensions apparaissent aussi bien dans les jugements interpersonnels que dans les stéréotypes à propos des groupes sociaux (Judd et al., 2005). Déjà en 1968, Rosenberg et ses collègues (Rosenberg, Nelson \& Vivekananthan, 1968) ont examiné la structure des jugements interpersonnels et ont montré qu'une représentation spatiale bidimensionnelle était la plus adéquate. Leurs résultats indiquent que les jugements interpersonnels sont sous-tendus par la dimension « intellectuelle » d'une part, et par la dimension « sociale » d'autre part. Asch (1946) a également montré l'importance des traits tels que « intelligent » et « chaleureux », autrement dit des traits liés soit à la dimension de compétence, soit à la dimension de sociabilité, dans la formation d'impression. Ces deux dimensions apparaissent aussi dans les jugements que les individus portent sur eux-mêmes. Ainsi, Tafarodi et Swann (1995) ont montré que l'estime de soi était composée, elle aussi, 
de deux dimensions : la valeur sociale d'une part (self-liking) et l'efficacité personnelle d'autre part (self-competence). Bien que l'appellation donnée à ces deux dimensions varie d'un auteur à l'autre, il s'agit bien du même contenu qui est étudié.

De la même manière, le contenu des stéréotypes a souvent été décrit sur base de ces deux dimensions de compétence et de sociabilité, ou moralité pour certains auteurs (voir par exemple Phalet \& Poppe, 1997 ; Poppe \& Linssen, 1999). En ce qui concerne les stéréotypes de genre, Spence et Helmreich (1978) ont avancé l'idée qu'ils se structuraient selon les dimensions d'expressivité, qui correspond à la sociabilité, et d'instrumentalité, qui correspond à la compétence. De manière consistante avec cette idée, Fiske et ses collègues ont étudié les stéréotypes décrivant de nombreux groupes sociaux et ont montré que les exogroupes se différenciaient généralement sur base des dimensions de compétence et de sociabilité (Fiske et al., 2002 ; Fiske et al., 1999). Fiske et ses collègues, ainsi que d'autres auteurs (voir par exemple Alexander et al., 1999 ; Conway, Pizzamiglio \& Mount, 1996), ont également montré que l'attribution de compétence aux membres d'un groupe était en grande partie déterminée par le statut relatif de ce groupe (dominant $v$ s. subordonné), tandis que l'attribution de sociabilité était principalement déterminée par le type de relation (coopérative $v s$. compétitive) que l'endogroupe entretient avec le groupe jugé. Ainsi, les membres des groupes dominants (haut statut) sont généralement perçus comme très compétents, tandis que les membres des groupes subordonnés (bas statut) sont souvent jugés peu compétents. Les membres des groupes sociaux avec lesquels nous coopérons sont généralement vus comme sociables et sympathiques, alors que les membres des groupes avec lesquels nous sommes en compétition sont fréquemment perçus comme peu sociables et froids. En résumé, l'étude du contenu des stéréotypes semble indissociable de celle de ces deux dimensions, compétence et sociabilité. La composante prescriptive des stéréotypes n'échappe pas à cette règle, comme en témoignent les études que nous décrirons dans cet article.

\section{L'AMBIVALENCE DES STÉRÉOTYPES}

Fiske et ses collègues $(1999 ; 2002)$ ont montré que les stéréotypes de nombreux groupes ont un contenu ambivalent, c'est-à-dire qu'ils combinent à la fois des caractéristiques négatives et des caractéristiques 
subjectivement positives. Cette ambivalence s'exprime sur les dimensions de sociabilité et de compétence que nous avons décrites plus haut. Ainsi, certains groupes sociaux sont perçus comme hautement compétents mais peu sociables (par exemple, les riches, les féministes, les femmes d'affaire...), et d'autres comme très sociables mais peu compétents (par exemple, les femmes au foyer, les personnes handicapées...). Les résultats de Fiske et al. (1999; 2002) indiquent que, pour de nombreux groupes sociaux, ces deux dimensions sont en effet corrélées négativement. De plus, l'aspect ambivalent des stéréotypes apparaît aussi bien dans les jugements émis à propos d'un exogroupe que dans les jugements à propos de l'endogroupe, peu importe le statut (dominant ou subordonné) des groupes. En effet, Yzerbyt, Provost et Corneille (2005), dans une étude sur les relations linguistiques entre les Français et les Belges, ont montré que leurs participants, quel que soit leur groupe d'appartenance, attribuaient un haut niveau de compétence et un bas niveau de sociabilité aux membres du groupe jugé de haut statut (les Français), tandis qu'ils attribuaient peu de compétence mais beaucoup de sociabilité aux membres du groupe jugé de bas statut (les Belges). Judd et ses collègues (Judd et al., 2005) obtiennent des résultats semblables en manipulant les informations données à propos d'un groupe social. Pour ce faire, ils décrivent deux groupes à leurs participants. Le premier est décrit à travers des comportements indiquant une compétence (ou sociabilité) élevée, tandis que le deuxième groupe est décrit par des comportements indiquant une faible compétence (ou sociabilité). Après avoir pris connaissance de ces informations, les participants évaluent les deux groupes sur une série de traits liés aux dimensions de compétence et de sociabilité. Leurs résultats indiquent qu'un groupe décrit comme hautement compétent est jugé moins sociable qu'un groupe décrit comme peu compétent. De même, lorsqu'un groupe est présenté comme étant très sociable, on lui attribue moins de compétence qu'à un groupe décrit comme peu sociable. De plus, les participants qui percevaient une grande différence entre les deux groupes sur une dimension (par exemple la compétence) percevaient également une différence importante sur l'autre dimension (par exemple la sociabilité). Ainsi, au travers de leurs inférences quant à la dimension sur laquelle ils n'ont pas reçu d'information, les individus expriment un certain degré d'ambivalence dans leurs évaluations des groupes. 
Selon Glick et Fiske (2001), les stéréotypes ambivalents permettent aux individus de légitimer l'utilisation de la composante négative de ces derniers dans leurs jugements sans pour autant sembler avoir une attitude entièrement hostile envers le groupe jugé, puisque des caractéristiques négatives (exemple : incompétent) sont «compensées» par des caractéristiques positives (exemple : sociable) qui sont attribuées en même temps aux membres d'un groupe. Ainsi, l'attribution de caractéristiques positives à l'exogroupe permet à l'individu de se défendre d'avoir des préjugés entièrement négatifs à propos de cet exogroupe.

Glick et Fiske (2001) avancent également l'idée selon laquelle les stéréotypes ambivalents peuvent être utilisés pour maintenir certains groupes dans une position subordonnée. En effet, selon ces auteurs, les traits de sociabilité correspondent à des comportements de respect et de déférence. Les stéréotypes qui décrivent les subordonnés comme sociables, chaleureux, et respectueux, refléteraient donc les attentes des dominants en ce qui concerne le comportement des subordonnés. Ainsi, le groupe dominant maintiendrait les subordonnés « à leur place » sans utiliser la force. Beaucoup de systèmes sociaux inégalitaires, comme par exemple les relations entre hommes et femmes, sont maintenus à travers un paternalisme dans lequel les membres du groupe subordonné sont stéréotypés comme sociables (Jackman, 1994) mais souvent peu compétents. Plusieurs auteurs pensent en effet que le sexisme, lorsqu'il correspond à une forme de paternalisme, est très efficace pour maintenir les femmes dans des statuts subordonnés (voir par exemple Benokraitis, 1997 ; Moya, Glick, Exposito, de Lemus et Hart, 2007), comme par exemple dans un emploi sous-payé ou dans le rôle traditionnel de femme au foyer. On sait en outre que les idéologies paternalistes ont un impact réel sur la performance des groupes subordonnés (Dardenne, Dumont \& Bollier, 2007). Nous reviendrons sur ce point plus tard.

En bref, il apparaît donc que les stéréotypes comprennent des caractéristiques issues des dimensions de sociabilité et de compétence et que les membres d'un groupe sont souvent décrits de manière ambivalente sur ces deux dimensions. Cette ambivalence légitimerait l'image et la position sociale de certains groupes. Certaines des caractéristiques de sociabilité ou de compétence attribuées aux membres d'un groupe peuvent en outre faire l'objet de prescriptions. Dans quelles situations ces caractéristiques sont-elles prescrites ? C'est la question que nous traiterons ci-après, en nous penchant plus particulièrement sur les stéréotypes sexuels. 


\section{RELATIONS INTERGROUPES ET STÉRÉOTYPES PRESCRIPTIFS}

Si certaines des caractéristiques censées être possédées par les subordonnés peuvent se révéler avantageuses pour les dominants, il semble logique que ces caractéristiques soient fortement valorisées par ces derniers. Ainsi, le stéréotype décrivant les subordonnés comme chaleureux, doux et respectueux deviendrait prescriptif afin de garantir l'avantage des dominants. Il réduirait ainsi la probabilité que les subordonnés ne se rebellent et ne tentent d'améliorer leur situation sociale, garantissant de ce fait le maintien du statu quo. Il s'agirait donc d'un moyen subtil, pour le groupe dominant, de contrôler le groupe subordonné sans utiliser la force, mais en employant un «gant de velours » (Jackman, 1994). Selon Glick et Fiske (2001), puisque, d'une part, le subordonné idéal respecte et se soumet au groupe dominant, et que, d'autre part, les traits de sociabilité correspondent à des comportements de respect et de soumission, on devrait observer un haut niveau de prescription sur les traits de sociabilité des subordonnés (Rudman \& Glick, 2001).

Dans le même ordre d'idée, il est probable que certaines caractéristiques, lorsqu'elles sont possédées par les subordonnés, représentent une menace pour les dominants. Ainsi, certains traits liés à la dimension de compétence pourraient être utilisés par les subordonnés afin d'obtenir davantage de pouvoir et/ou un statut plus élevé. Par exemple, des subordonnés qui se montrent ambitieux, compétitifs et bien organisés seraient plus susceptibles de menacer la position avantageuse des dominants que des subordonnés qui se montrent dociles, soumis et mal organisés. Par conséquent, il est probable que, afin de maintenir leur avantage, les dominants ne se contentent pas de prescrire aux subordonnés des traits liés à la sociabilité, mais leur proscrivent également toute une série de traits liés à la compétence.

Les relations traditionnelles entre hommes et femmes suivent ce schéma de relation entre dominants et subordonnés. De plus, une certaine forme de dépendance des hommes par rapport aux femmes (comme partenaires romantiques, pour la reproduction, ou encore pour l'éducation des enfants) inciterait les hommes à maintenir les femmes dans un rôle subordonné, tout en entretenant une relation de coopération (Rudman \& Glick, 2001). Les hommes prescriraient donc aux femmes de nombreux traits de sociabilité, puisque ces derniers correspondent à des comportements de soumission et de respect. À l'inverse, les traits de compétence seraient peu prescrits (voire proscrits) aux femmes, tandis qu'ils seraient prescrits aux hommes. Prentice et Carranza (2002) ont directement testé cette hypothèse 
en demandant à leurs participants d'indiquer dans quelle mesure il était souhaitable de posséder une série de traits soit pour un homme, soit pour une femme, ou encore pour une personne en général. Lorsqu'un trait était jugé plus souhaitable pour une femme que pour une personne en général, les auteurs considéraient qu'il était prescrit aux femmes. Si le trait était plus souhaitable pour un homme que pour une personne en général, ils considéraient alors que le trait était prescrit aux hommes. Les résultats de cette étude ont indiqué que les femmes se voyaient effectivement prescrire des traits de sociabilité (tels que chaleureuse, gentille, coopérative, patiente et polie), tandis que des traits de compétence étaient prescrits aux hommes (comme, par exemple, ambitieux, assertif, confiant, rationnel et compétitif). Le même type de résultats a été observé dans d'autres études en ce qui concerne les émotions prescrites aux hommes et aux femmes. Il a en effet été montré que les femmes se voyaient prescrire davantage d'émotions indiquant la subordination et la sociabilité, telles que la crainte, tandis que les hommes se voyaient prescrire des émotions indiquant plutôt le pouvoir et la dominance, comme par exemple la colère (Hess, Adams \& Kleck, 2005, voir aussi Timmers, Fischer \& Manstead, 2003). Les résultats de ces différentes études ne révèlent aucun effet du sexe des participants. En d'autres termes, il apparaît que les hommes et les femmes prescrivent généralement le même contenu. Néanmoins, une étude de Timmers et al. (2005) indique que les hommes expriment leur accord avec les prescriptions de genre davantage que les femmes. Cette plus forte tendance prescriptive des hommes est en ligne avec l'hypothèse selon laquelle la prescription leur serait bénéfique. Alors que les causes de la prescription appliquée par les dominants aux subordonnés sont facilement compréhensibles, les raisons qui pousseraient les subordonnés à adhérer de la même façon à ces prescriptions semblent moins évidentes. Pourquoi les membres des groupes subordonnés adhèrent-ils si souvent à des prescriptions susceptibles de les maintenir dans une position désavantageuse ? Pourquoi cautionnent-ils des prescriptions qui favorisent un autre groupe que le leur?

\section{LA FONCTION DE JUSTIFICATION DU SYSTÈME DES STÉRÉOTYPES}

On sait non seulement que les membres de groupes subordonnés acceptent et véhiculent souvent des prescriptions stéréotypiques allant à l'encontre 
des intérêts de leur groupe, mais on sait également que, lorsque certains membres d'un groupe, quel que soit son statut, ne se conforment pas aux attentes stéréotypiques, ils sont jugés négativement aussi bien par les membres des autres groupes que par les membres de leur groupe (voir, par exemple, Jussim, Coleman \& Lerch, 1987). Il a par exemple été montré que même les femmes sanctionnent d'autres femmes qui ne se conforment pas aux attentes stéréotypiques. Plusieurs études ont en effet indiqué que les participantes féminines exprimaient un jugement aussi négatif que les participants masculins face à une femme qui ne se conformait pas aux prescriptions de genre (Heilman \& Okimoto, 2007 ; Heilman, Wallen, Fuchs \& Tamkins, 2004). Ainsi, il apparaît que les prescriptions stéréotypiques sont également véhiculées et maintenues par ceux qui en sont la cible.

La Théorie de la Justification du Système (Jost \& Banaji, 1994) peut nous aider à mieux comprendre pourquoi les membres des groupes subordonnés adhèrent aux stéréotypes qui leur confèrent une haute sociabilité mais peu de compétence, et par conséquent ne favorisent pas leur groupe au niveau sociétal. De plus, cette théorie peut nous éclairer sur la relation entre prescription et maintien du système social. La Théorie de la Justification du Système a été développée pour expliquer une des fonctions essentielles des stéréotypes ; celle de légitimation. Selon Jost \& Banaji (1994), les stéréotypes peuvent servir trois types de légitimation (voir aussi Jost, Burgess \& Mosso, 2001 ; Jost \& Hunyady, 2002). Tout d'abord, ils peuvent être utilisés à des fins de légitimation personnelle (ego-justification). Dans ce cas, les individus utilisent le stéréotype pour défendre et légitimer leur propre position dans la société. En second lieu, les stéréotypes peuvent avoir pour fonction la légitimation du groupe. Jost et Banaji (1994) développent cette fonction en s'appuyant sur l'idée de Tajfel (1981) selon laquelle les stéréotypes serviraient à légitimer les attitudes et les actions de l'endogroupe envers un/des exogroupe(s). Les stéréotypes permettraient également de maintenir une bonne estime de soi grâce à une identité groupale positive. Ces deux premières fonctions ne permettent cependant pas d'expliquer le phénomène d'auto-stéréotypisation négative (voir par exemple Sagar \& Schofield, 1980) ni l'auto-prescription de caractéristiques désavantageuses chez les groupes subordonnés. Par contre, la troisième fonction des stéréotypes, la justification du système, permet d'expliquer ces phénomènes. Selon Jost et Banaji (1994), un des rôles des stéréotypes serait d'expliquer et de légitimer le système social existant et les positions relatives des différents groupes sociaux, avec comme résultat le maintien de ce système social. Les individus, quel que soit leur groupe d'appartenance, seraient motivés à croire que le système dans lequel ils vivent est juste 
et légitime (Jost, Pelham, Sheldon \& Sullivan, 2003 ; Rubin \& Peplau, 1975). En attribuant une haute compétence aux groupes dominants et une faible compétence aux groupes subordonnés, ils expliquent et légitiment la position relative de chaque groupe dans la société. En prescrivant une haute compétence aux dominants d'une part et une haute sociabilité ainsi qu'une faible compétence aux subordonnés d'autre part, ils assurent la stabilité du système social en place. Plusieurs études ont ainsi montré que les membres des groupes dominants n'étaient pas les seuls à défendre le système social en place, mais que les membres des groupes subordonnés soutiennent également le système social (voir par exemple Jost et al., 2003) et expriment du favoritisme envers le groupe dominant (voir par exemple Jost, Pelham \& Carvallo, 2002). En somme, selon la Théorie de la Justification du Système, la composante prescriptive des stéréotypes se développerait afin de maintenir le système social en place. Les individus, quel que soit le statut de leur groupe d'appartenance, adhéreraient à ces stéréotypes prescriptifs car ils leur permettent de comprendre et justifier le système dans lequel ils vivent.

$\mathrm{Si}$, comme nous l'avons proposé, les traits et les émotions prescrits aux membres des groupes dominants et subordonnés permettent de maintenir le statu quo et, de ce fait, la position avantageuse des dominants, alors il est hautement probable que l'individu qui ne se conforme pas à ces prescriptions soit fortement sanctionné. En effet, ce dernier, en violant les attentes stéréotypiques, menace l'équilibre du système social et le statut des dominants. En adhérant aux prescriptions, les membres des groupes subordonnés recueillent également un bénéfice : celui d'éviter la sanction.

\section{VIOLATION DES PRESCRIPTIONS STÉRÉOTYPIQUES ET DISCRIMINATION}

La discrimination liée à la composante prescriptive d'un stéréotype concerne les membres d'un groupe qui ne se conforment pas aux prescriptions dont leur groupe fait l'objet (Heilman, 2001). Par exemple, il a plusieurs fois été montré qu'une femme qui ne se conforme pas aux attentes traditionnelles (qui ne se montre pas sensible et maternelle par exemple) est la cible de comportements hostiles (voir Burgess \& Borgida, 1999 ; Eagly \& Karau, 2002 ; Gill, 2004 ; Rudman \& Glick, 1999, 2001). Dans cette 
situation, la discrimination deviendrait donc un moyen de sanctionner la violation des prescriptions stéréotypiques.

On sait que les normes injonctives, c'est-à-dire les normes qui spécifient comment les individus doivent se comporter (ce qui correspond à de la prescription), motivent le comportement par le biais de récompenses à ceux qui s'y conforment ou de sanctions sociales à ceux qui refusent de s'y plier (Cialdini \& Trost, 1998). Par ailleurs, il a été montré que les individus qui ne se conforment pas aux normes de leur groupe souffrent de diverses conséquences négatives. Ils sont souvent peu appréciés, on leur assigne des emplois de statut subordonné, ils sont victimes de pressions à la conformité et, dans certains cas, ils sont exclus de leur propre groupe (voir par exemple Manstead, Hewstone, Fiske, Hogg, Reis \& Semin, 1995). De nombreuses études ont montré que les femmes qui ne se conforment pas aux stéréotypes traditionnels, c'est-à-dire celles qui ne se montrent pas à la fois sociables et peu compétentes, sont effectivement victimes de sanctions et de discrimination. Par exemple, on sait que les femmes qui se comportent d'une manière autoritaire sont évaluées plus négativement qu'un homme se comportant de la même manière (Eagly, Makhijani \& Klonsky, 1992). De même, il a été montré qu'une femme qui refuse de poser un comportement altruiste est jugée plus négativement qu'un homme dans la même situation (Heilman \& Chen, 2005). Les femmes elles-mêmes s'attendent à des sanctions sociales si elles n'expriment pas des émotions positives envers les autres (Stoppard \& Gunn Gruchy, 1993).

Dans une revue de la littérature, Heilman (2001) présente plusieurs études indiquant que, même dans un cadre professionnel, les femmes hautement compétentes sont souvent critiquées et peu aimées. Elles peuvent se voir refuser des promotions ou des postes de haut statut qui sont accordés à des hommes de compétence identique. Récemment, Heilman, Wallen, Fuchs et Tamkins (2004) ont montré à travers trois études que, à compétence égale, une femme occupant un emploi stéréotypiquement masculin était moins appréciée et davantage critiquée qu'un homme dans le même emploi. Leurs résultats indiquent en outre que l'image négative dont souffre la femme compétente peut affecter ses chances de promotion à un poste de plus haut statut. Rudman et Glick (2001) ont également montré qu'une femme qui postule à un emploi stéréotypiquement féminin et qui met en avant ses qualités de compétence était moins engagée qu'un autre candidat car elle était perçue comme peu sensible et peu sociable. De même, des études récentes (Heilman \& Okimoto, 2007) ont montré qu'une femme qui se montre très compétente dans un emploi stéréotypiquement masculin était jugée plus négativement que sa contrepartie masculine. La cible féminine était moins appréciée, était jugée plus hostile et était davantage 
rejetée comme patron potentiel que la cible masculine. Cependant, lorsque les participants des études recevaient de l'information indiquant que la cible était sociable et sensible en plus d'être compétente, ils évaluaient la cible féminine de la même manière que la cible masculine. Ces résultats suggèrent que la réaction négative face à une femme compétente dans un domaine masculin est causée par la perception d'une violation des prescriptions de genre. Dans les études de Heilman et Okimoto (2007), le simple fait de fournir des informations stéréotypiques à propos de la cible (par exemple, mentionner qu'elle a des enfants) permet d'atténuer l'effet de la violation du stéréotype. Malheureusement, le fait de fournir de l'information stéréotypique à propos d'une cible contre-stéréotypique ne permet pas toujours d'éviter la discrimination. Cuddy, Fiske et Glick (2004) ont en effet montré que les femmes professionnellement actives et décrites comme ayant un enfant étaient jugées plus sociables mais moins compétentes que leurs homologues sans enfant. Il semble donc que, lorsque les femmes professionnellement actives deviennent mères, bien qu'elles gagnent en sociabilité perçue, elles perdent en compétence perçue. À l'inverse, les hommes décrits comme ayant des enfants sont perçus comme plus sociables que leurs homologues sans enfant, mais tout aussi compétents. De plus, les femmes avec enfant(s) sont plus susceptibles d'être victimes de discrimination professionnelle (par exemple à l'embauche ou pour les promotions) que les femmes sans enfant, tandis que cette différence n'apparaît pas entre les hommes avec et sans enfant(s). En somme, il apparaît, que le fait de fournir des informations stéréotypiques à propos d'une cible n'a pas toujours des conséquences bénéfiques pour cette dernière.

Bien que la plupart des études sur les conséquences des violations stéréotypiques se concentrent sur le stéréotype de la femme, certaines recherches, bien que moins nombreuses, indiquent que les hommes n'échappent pas aux sanctions s'ils ne se conforment pas aux stéréotypes traditionnels. Ainsi, Rudman et Glick (1999) ont montré que les hommes décrits en termes stéréotypiquement féminins (par exemple, tournés vers les relations interpersonnelles) étaient perçus comme très sociables mais comme moins compétents que des hommes décrits en termes stéréotypiquement masculins (par exemple, tourné vers la prise de décisions). Ils étaient également moins recommandés pour un emploi que leur contrepartie plus «masculine ». Dans une étude plus récente, Rudman et Fairchild (2004) ont demandé à leurs sujets de participer à un jeu sur ordinateur lors duquel ils étaient en compétition avec un concurrent homme ou femme. Ce concurrent était en réalité un comparse de l'expérimentateur. À l'issue du jeu, les participants perdaient et le comparse 
gagnait. Le jeu consistait en une tâche soit stéréotypiquement masculine (sur le thème du football), soit stéréotypiquement féminine (sur le thème du développement infantile). Dans la moitié des cas, les participants se retrouvaient donc face à un homme ou une femme contre-stéréotypique, puisque ce dernier/cette dernière excellait dans une tâche stéréotypique de l'autre sexe. À l'issue de cette compétition et de l'échec des participants, il leur était donné la possibilité de saboter la performance du comparse, en préparant la tâche suivante que ce dernier devrait effectuer. Il est apparu que les participants, peu importe leur sexe, ont davantage saboté la performance des concurrents contre-stéréotypiques, quel que soit le sexe de ceux-ci, que celle des concurrents stéréotypiques. Autrement dit, les hommes contre-stéréotypiques, c'est-à-dire ceux qui adoptaient des caractéristiques typiquement féminines, étaient discriminés au même titre que les femmes contre-stéréotypiques.

La recherche en psychologie développementale a également révélé une attitude négative envers les garçons qui adoptent des comportements perçus comme typiques de l'autre sexe. Par exemple, Sandnabba et Ahlberg (1999) ont montré que les petits garçons qui ont des comportements jugés comme féminins étaient évalués plus négativement que les petites filles qui adoptent des comportements typiquement masculins. De plus, les participants à cette étude s'attendaient à ce que les petits garçons féminins soient moins bien adaptés psychologiquement dans leur vie future que les enfants plus stéréotypiques de leur sexe. Enfin, on sait que les hommes homosexuels, qui violent certaines prescriptions de genre, sont aujourd'hui encore victimes de discrimination et cibles d'attitudes négatives (voir par exemple Yang, 1997). Ces résultats semblent donc indiquer que les hommes qui violent les prescriptions de genre sont eux aussi jugés négativement et victimes de sanctions, parfois même plus que les femmes. Cependant, il existe encore peu d'études sur la violation des prescriptions de genre chez les hommes et ses conséquences. Il est donc trop tôt pour conclure que ces conséquences sont similaires pour les hommes et pour les femmes.

En ce qui concerne les femmes, les conséquences négatives des violations de prescriptions sont, depuis quelques années, l'objet d'une attention croissante. Aux États-Unis, la discrimination fondée sur la composante prescriptive des stéréotypes de genre a même été reconnue d'un point de vue légal. Ainsi, en 1989, la recherche sur les stéréotypes de genre a fortement influencé la décision de la Court Suprême dans un procès opposant Ann Hopkins, une comptable, à Price Waterhouse, la compagnie pour laquelle elle travaillait (Fiske, Bersoff, Borgida, Deaux et Heilman, 1991). Cette compagnie avait refusé de promouvoir Hopkins comme associée en invoquant le prétexte de son manque de féminité et de ses 
problèmes relationnels. Pourtant, Hopkins était parmi les comptables les plus performant(e)s de la compagnie et était reconnue comme telle par ses supérieurs et ses clients. On lui attribuait des qualités de compétence, d'ambition et de ténacité. Ce sont précisément ces qualités, stéréotypiquement masculines, qui ont conduit Hopkins à être vue comme non conforme à l'image traditionnelle de la femme. Son comportement était perçu comme inapproprié pour une femme et il semble que la discrimination professionnelle dont elle a fait l'objet était une façon de la pénaliser pour ne pas avoir respecté les prescriptions des stéréotypes de genre. À l'issue de ce procès, le juge a déclaré : « un employeur qui traite une femme avec une personnalité assertive d'une manière différente que si elle avait été un homme est coupable de discrimination sexuelle » (cité dans Fiske et al., 1991), reconnaissant ainsi de manière officielle la discrimination sexuelle fondée sur la composante prescriptive des stéréotypes.

En résumé, les différentes études que nous venons de citer indiquent clairement que les femmes qui se montrent peu sociables et/ou hautement compétentes, autrement dit en contradiction avec le stéréotype, sont sanctionnées. Bien qu'il existe moins d'études à ce propos, il semble que les hommes qui dérogent aux attentes stéréotypiques seraient également victimes de sanctions. Il apparait donc que, lorsque des femmes (et, au moins dans certains cas, des hommes) manifestent des comportements ou caractéristiques contraires aux stéréotypes de genre, des sanctions sociales leur sont appliquées. Ceci traduit la volonté prescriptive liée aux stéréotypes. La conformité ou le respect des contenus stéréotypiques est recherché et désiré, et la déviance par rapport à ceux-ci est sanctionnée. Cependant, aucune des études mentionnées ne mesure vraiment la tendance prescriptive des participants jugeant les femmes ou les hommes contre-stéréotypiques. On ne peut donc pas déterminer avec certitude que la sanction est effectivement le résultat direct de cette volonté prescriptive contrariée. Pour pouvoir établir l'existence d'un lien entre violation des prescriptions de genre et sanction, il parait important de mesurer le niveau de volonté prescriptive afin de déterminer la force du lien qui les unit. À notre connaissance, Gill (2004) est le seul auteur qui ait étudié de manière directe et empirique ce lien entre prescription et discrimination. Pour ce faire, cet auteur a, dans un premier temps, mesuré l'adhésion de ses participants aux stéréotypes descriptif et prescriptif des femmes, en leur présentant une liste de traits de sociabilité et de compétence. Les participants devaient tout d'abord indiquer dans quelle mesure une femme " moyenne » possédait chacun de ces traits (aspect descriptif). Ensuite, il leur était demandé d'indiquer dans quelle mesure une femme idéale devait posséder ces traits (aspect prescriptif). Les participants 
répondaient sur une échelle de Likert dont les extrémités étaient étiquetées « moins qu'un homme » et «plus qu'un homme ». Lorsque, pour les participants, une femme devait posséder un trait plus qu'un homme, alors ce trait était considéré comme prescriptif. Environ un mois après avoir complété ce premier questionnaire, les participants étaient confrontés à une description d'emploi et aux curriculum vitae d'un homme et d'une femme de qualifications égales. Ils réalisaient alors une tâche de décision d'embauche. Les résultats de Gill (2004) indiquent que l'adhésion des participants au stéréotype prescriptif de la femme prédit un biais dans le choix du/de la candidat(e). Les participants ayant une forte tendance prescriptive choisissaient significativement moins souvent la candidate que le candidat. L'adhésion au stéréotype descriptif, quant à elle, n'était liée à aucun biais.

\section{PRESCRIPTION ET MAINTIEN DE L'AVANTAGE DES DOMINANTS}

Il apparaît, à la lumière des études que nous avons présentées, que les membres du groupe subordonné (dans la plupart de ces études, les femmes) se voient prescrire des traits de sociabilité (et éventuellement proscrire des traits de compétence), tandis que les membres du groupe dominant (en général, les hommes) se voient plutôt prescrire de la compétence, et ce aussi bien par les membres de l'endogroupe que par les membres de l'exogroupe. De plus, les études présentées indiquent que les individus qui ne se conforment pas aux prescriptions sont susceptibles d'être sanctionnés. On peut dès lors penser que les stéréotypes prescriptifs liés au genre servent les avantages des hommes, puisque la prescription de sociabilité (et la proscription de compétence) aux femmes restreint l'accès de celles-ci à des positions de haut statut. De plus, il a été montré que certaines idéologies paternalistes, qui prescrivent de la sociabilité et proscrivent de la compétence aux femmes, peuvent objectivement altérer les performances de ces dernières. Concrètement, Dardenne et ses collègues (2007) ont demandé à leurs participantes de se mettre dans la peau de candidates prenant part à un test de recrutement pour une entreprise. Certaines d'entre elles étaient placées dans un contexte sexiste bienveillant, tandis que les autres participantes étaient placées soit dans un contexte sexiste hostile, soit dans un contexte non-sexiste. Le sexisme bienveillant 
correspond à une attitude subjectivement positive envers les femmes, mais qui sous-entend une moindre compétence de celles-ci et qui leur attribue un statut subordonné à celui des hommes. Le sexisme hostile renvoie, comme son nom l'indique, à une attitude ouvertement sexiste et négative envers les femmes. La performance des participantes à un test de résolution de problèmes était ensuite mesurée. Les résultats ont révélé que la performance des femmes placées dans un contexte sexiste bienveillant était significativement moins bonne que celle des candidates placées dans les conditions sexiste hostile et contrôle. De plus, il est apparu que la moins bonne performance des participantes était causée par des pensées intrusives reflétant des doutes à propos de leur propre compétence. Ces résultats montrent que les idéologies paternalistes et les prescriptions qu'elles véhiculent peuvent effectivement mener les femmes à douter de leur compétence et à avoir de moins bonnes performances (voir aussi Adams, Garcia, Purdie-Vaughns \& Steele, 2006).

Non seulement, un contexte paternaliste peut avoir un impact sur la performance des femmes, mais le simple rappel du stéréotype de moindre compétence des femmes peut suffire à altérer leurs performances dans certains types de tâches. Ainsi, les études sur la menace du stéréotype ont montré que les femmes ont une moins bonne performance à un test de mathématiques que les hommes lorsqu'elles pensent que ce test a révélé par le passé des différences liées au sexe (Spencer, Steele \& Quinn, 1999). Davies, Spencer et Steele (2005) ont également montré que le simple fait de rappeler à leurs participantes le stéréotype de la femme les menait ensuite à refuser un rôle de leader au profit d'un rôle de subordonné. D'autre part, il arrive souvent que le stéréotype d'incompétence soit intériorisé par les femmes, et ce dès le plus jeune âge. Par exemple, Herbert et Stipek (2005) ont montré que des petites filles de 8 ans évaluaient leurs compétences en mathématiques plus négativement que des garçons du même âge, alors qu'il n'y avait en réalité aucune différence objective au niveau de la réussite dans ce domaine. L'intériorisation du stéréotype décrivant les femmes comme plus sociables mais moins compétentes que les hommes a pour conséquence de mener celles-ci à éviter certains domaines ou certains rôles perçus comme plus stéréotypiques des hommes (voir par exemple Eccles, 2007 ; Dickhäuser et Stiensmeier-Pelster, 2002). Cette intériorisation du stéréotype est par conséquent susceptible de freiner l'accès des femmes à un statut plus élevé.

Les stéréotypes sexuels ainsi que les idéologies qui prescrivent de la sociabilité (et proscrivent la compétence) aux femmes pourraient donc avoir pour conséquence le maintien des femmes dans un statut subordonné. De plus, dans la mesure où ces dernières se conforment à la prescription 
de sociabilité dont elles font l'objet, elles favorisent une relation de coopération avec les hommes, en se conduisant de façon sociable et déférente (Glick \& Fiske, 2001). De la même façon, la prescription de compétence aux hommes, si ceux-ci s'y conforment, devrait leur assurer un statut élevé. Néanmoins, rien ne permet de dire avec certitude que les résultats décrits jusqu'à présent sont dus à la différence de statut entre les deux groupes. Ces résultats ne permettent pas non plus d'affirmer que la prescription de sociabilité aux femmes a pour fonction de servir les avantages des hommes. Les effets observés pourraient tout à fait être liés aux rôles spécifiques que les femmes et les hommes occupent dans le système social et pas à leurs statuts respectifs.

Une étude de Hess et ses collègues (2005) indique cependant clairement que la composante prescriptive des stéréotypes est bien liée à la différence de statut entre les dominants et les subordonnés. Dans le domaine des émotions, les hommes et les femmes se voient prescrire des caractéristiques très différentes. En général, on prescrit aux hommes des émotions liées au pouvoir (la colère par exemple) tandis qu'on prescrit aux femmes des émotions liées à la sociabilité et la soumission (par exemple la crainte ou la joie). Hess et al. (2005) ont avancé l'idée que le type d'émotions prescrites était en réalité lié au statut des individus et pas directement à leur genre. Pour tester cette hypothèse, ils ont montré à leurs participants des photos de visages d'hommes et de femmes, neutres d'un point de vue émotionnel. Pour chacune des photos, ils ont demandé à un premier groupe de participants d'indiquer à quelle fréquence la personne présentée était susceptible de manifester certaines émotions spécifiques (par exemple, la colère, la joie, la surprise, ou encore la tristesse). Il était demandé à un deuxième groupe de participants d'indiquer leur perception du niveau de dominance sociale de chacune des personnes photographiées. Enfin, un troisième et dernier groupe de participants indiquaient leur perception du niveau de soumission de chacune des personnes prises en photo. Les auteurs ont calculé, pour chacune des photos, les scores moyens de dispositions émotionnelles, de dominance perçue et d'affiliation (ou soumission) perçue. Ils ont ensuite analysé les relations entre ces trois variables et le genre des personnes présentées sur les photos dans une analyse de médiation pour laquelle les unités d'analyses étaient chacun des visages photographiés. Hess et al. (2005) ont ainsi montré que le sexe de la cible prédit le type d'émotions prescrites, mais que cette relation n'est pas directe. La perception de dominance/soumission est un médiateur de cette relation. Il apparaît que les hommes sont généralement perçus comme plus dominants tandis que les femmes sont vues comme plus soumises. Les individus perçus comme dominants se voient prescrire des émotions liées 
au pouvoir, tandis que les individus perçus comme subordonnés se voient prescrire des émotions liées à la soumission et à la sociabilité.

Toujours en ce qui concerne les émotions, il a été montré que les hommes ont une plus forte tendance prescriptive que les femmes. Ainsi, Timmers et ses collègues (2003) ont demandé à leurs participants de répondre à une série de questions évaluant leurs croyances descriptives et prescriptives à propos des réactions émotionnelles des hommes et des femmes. Les items sur les croyances prescriptives consistaient en des évaluations négatives ou des désapprobations de réactions émotionnelles contre-stéréotypiques chez des hommes ou chez des femmes (par exemple, « les hommes qui expriment de la peur sont faibles» ou « les femmes ne devraient pas montrer leur colère »). Ces items se répartissaient en fait en deux sous-échelles : la première évaluait la désapprobation de manifestations d'impuissance ou de sensibilité, tandis que la deuxième évaluait la désapprobation de manifestations de pouvoir. Les résultats de cette étude ont indiqué que les hommes exprimaient davantage leur accord avec les items de l'échelle prescriptive que les femmes. On peut en conséquence faire l'hypothèse que, si les hommes adhèrent davantage aux prescriptions de genre que les femmes, c'est parce que ces prescriptions leur sont plus avantageuses qu'aux femmes. Néanmoins, il ne s'agit là que d'une supposition et l'étude de Timmers et al. (2003) ne nous permet pas de tirer une telle conclusion de manière définitive. Plusieurs études peuvent cependant nous éclairer sur ce lien entre aspect avantageux des traits et prescription. Diekman et Goodfriend (2006) ont présenté à leurs participants la description d'un groupe inconnu de ces derniers. Le pays dans lequel vivait ce groupe, la Moldavie, était décrit comme ayant une politique d'emploi mettant fortement l'accent sur la compétitivité pour la moitié des participants. Dans le texte que lisait l'autre moitié des participants, la Moldavie était décrite comme un pays favorisant les relations interpersonnelles dans sa politique d'emploi. Ensuite, les auteurs évaluaient la mesure dans laquelle une série de traits de compétence et de sociabilité étaient perçus par les participants comme utiles pour le Moldave moyen. Enfin, ils évaluaient la mesure dans laquelle les participants prescrivaient les mêmes traits de compétence ou de sociabilité aux habitants du pays. Il est apparu que la prescription des traits (de compétence ou de sociabilité) variait selon le contexte politique. Lorsque la politique du pays favorisait la compétitivité, davantage de traits de compétence étaient prescrits. Tandis que lorsque les relations interpersonnelles étaient favorisées, ce sont principalement des traits de sociabilité qui étaient prescrits. Néanmoins, cette relation entre le contexte politique et la prescription n'était pas directe. L'évaluation de l'utilité des 
traits était un médiateur de cette relation. Donc, selon le contexte, la compétence/sociabilité était jugée plus ou moins utile chez les habitants de la Moldavie. Plus elle était perçue comme utile, plus elle était prescrite. On pourrait conclure que ces résultats sont en ligne avec notre hypothèse, évoquée plus haut, à savoir que les membres des groupes dominants vont prescrire des traits aux subordonnés dans la mesure où ces traits sont susceptibles de leur apporter des bénéfices. Cependant, dans l'étude de Diekman et Goodfriend (2006), l'utilité des traits n'est pas mesurée en référence à une relation dominant-subordonné, puisqu'ils demandent à leurs participants d'indiquer dans quelle mesure chacun des traits est utile pour le Moldave moyen. Cette mesure n'évalue pas si le trait est perçu comme plutôt avantageux pour le(s) groupe(s) dominant(s), plutôt avantageux pour le(s) groupe(s) subordonné(s), ou avantageux pour tous. De plus, la mesure de prescription utilisée par ces auteurs est critiquable. La prescription d'un trait est évaluée en demandant aux participants de juger la positivité de chaque trait possédé par le Moldave moyen. Cette mesure semble donc être une mesure de valence plutôt qu'une mesure de prescription. Bien que très intéressants pour notre propos, les résultats de cette étude sont donc à interpréter avec précaution.

Dans un ensemble d'études récentes, nous (Delacollette, Dumont et Dardenne, 2010 ; voir également Delacollette, Dardenne et Dumont, 2010) avons testé l'hypothèse selon laquelle l'aspect avantageux des traits pour le groupe dominant détermine bien la mesure dans laquelle ils sont prescrits aux membres du groupe subordonné. Pour ce faire, nous avons demandé à nos sujets de lire un scénario décrivant la vie de deux groupes fictifs, un groupe dominant et un groupe subordonné, sur une planète imaginaire. Dans une étude préliminaire, nous avons demandé aux participants d'indiquer, sur une échelle de Likert, dans quelle mesure une série de traits, lorsqu'ils étaient possédés par les membres du groupe subordonné, étaient avantageux pour les membres du groupe dominant. Nous avons ensuite calculé pour chacun de ces traits un score moyen de bénéfice. Nous avons comparé ce score de bénéfice au point central de l'échelle de réponse. Les traits dont le score était supérieur à ce point central étaient considérés comme avantageux pour les dominants, tandis que les traits dont le score était égal ou inférieur à ce point central étaient considérés comme non-avantageux pour le groupe dominant. Cette étude préliminaire nous a ainsi permis de mettre en évidence différentes catégories de traits selon leur aspect (plus ou moins) avantageux pour les dominants. Ensuite, dans deux études, il était demandé aux participants de se mettre dans la peau soit d'un membre du groupe dominant soit d'un membre du groupe subordonné et de se former une impression du groupe subordonné. Puis 
les participants répondaient à une série de questions évaluant la mesure dans laquelle les différents types de traits étaient prescrits. Les résultats de ces études ont indiqué que les membres du groupe subordonné se voyaient prescrire plus de traits avantageux pour le groupe dominant que de traits non avantageux. En effet, quel que soit le groupe auquel ils avaient été assignés, les participants prescrivaient aux subordonnés des traits liés à la dimension de sociabilité tels que chaleureux, altruistes et gentils, ainsi que des traits liés à la dimension de compétence tels que travailleurs, efficaces et habiles, mais uniquement lorsque ces derniers étaient perçus comme avantageux pour les dominants. À l'inverse, les subordonnés se voyaient prescrire très peu de traits tels que créatifs, organisés et ambitieux, jugés non-avantageux (voire menaçant) pour les dominants. La seconde étude a cependant révélé que ce pattern de résultats n'apparaissait que lorsque la différence de statut entre les deux groupes était décrite comme stable. Dans cette étude, nous avons manipulé la stabilité perçue de la relation dominants-subordonnés en utilisant deux versions du scénario. La première version était identique à celle utilisée dans les études 1 et présentait une situation intergroupe stable, dans laquelle la différence de statut entre les groupes était incontestée. La deuxième version du scénario décrivait une situation instable. Pour ce faire, nous avons ajouté au scénario utilisé dans les deux premières études un court paragraphe expliquant qu'un petit groupe de subordonnés n'acceptait plus de coopérer, réclamait davantage de respect et de pouvoir et tentait de convaincre d'autres subordonnés de se joindre à eux. Les résultats de cette troisième étude ont répliqué les résultats des études 1 dans la condition stable, autrement dit, lorsque les subordonnés étaient présentés comme travaillant en coopération avec les dominants sans revendiquer un statut plus élevé. Par contre, lorsque la différence de statut entre les groupes était décrite comme instable et que, par conséquent, les subordonnés représentaient une menace potentielle pour les dominants, alors l'ensemble des traits liés à la compétence étaient beaucoup moins prescrits. Dans une telle situation, les subordonnés sont en effet susceptibles d'utiliser leur compétence à leur propre avantage plutôt qu'au profit des dominants. Il est dès lors compréhensible que ces derniers leur prescrivent nettement moins de traits liés à la compétence. Ainsi, les résultats de ces études indiquent effectivement que les traits prescrits aux membres des groupes subordonnés sont généralement des traits utiles aux dominants, soutenant de ce fait l'idée selon laquelle la prescription serait un moyen de maintenir l'avantage de ces derniers.

Nous avons également montré que la prescription de sociabilité aux femmes est, elle aussi, déterminée par le bénéfice que cette sociabilité peut amener au groupe dominant, c'est-à-dire les hommes (Delacollette et al., 
2010). Elle est, de plus, dépendante du contexte. Nous avons demandé à des participants de sexe masculin de penser aux femmes, soit dans un contexte familial, soit dans un contexte professionnel. Puis nous leur avons demandé d'indiquer dans quelle mesure une série de traits liés à la sociabilité sont avantageux pour les hommes lorsqu'ils sont possédés par les femmes soit dans le cadre de leur travail (pour la moitié des participants) soit dans le cadre familial (pour le reste des participants). Ces traits avaient été identifiés par des études antérieures comme étant généralement prescrits aux femmes (Gill, 2004 ; Prentice \& Carranza, 2002). Ensuite, les participants indiquaient dans quelle mesure une femme idéale devait posséder chacun de ces traits plus qu'un homme ou moins qu'un homme (à nouveau soit dans le cadre de son travail, soit dans le cadre familial). Cette mesure constituait notre mesure de prescription. Enfin les participants complétaient l'Echelle de Sexisme Ambivalent (Dardenne, Delacollette, Grégoire \& Lecocq, 2006), une adaptation française de l'Ambivalent Sexism Inventory (Glick \& Fiske, 1996). Cette échelle évalue deux dimensions distinctes (mais corrélées) du sexisme : d'une part le sexisme hostile et, d'autre part, le sexisme bienveillant. Les résultats de cette quatrième étude indiquent que le contexte (familial ou professionnel) influence la perception du bénéfice que la sociabilité des femmes représente pour les hommes. À son tour, le bénéfice perçu détermine la mesure dans laquelle les traits de sociabilité sont prescrits aux femmes. Concrètement, les hommes perçoivent un bénéfice plus important dans les traits de sociabilité des femmes, tels que sensible, chaleureuse et patiente, dans un contexte familial que dans un contexte professionnel. Plus ils perçoivent ces traits comme avantageux, plus ils les prescrivent aux femmes. De plus, cette étude a indiqué que la composante bienveillante du sexisme influence également la perception du bénéfice. Les résultats de notre étude ont ainsi montré que l'adhésion au sexisme bienveillant menait à percevoir davantage la sociabilité des femmes comme avantageuse pour les hommes et, par conséquent, à la prescrire à ces dernières ${ }^{1}$. En somme, les résultats de cette étude montrent que le contexte intergroupe influence bel et bien la prescription de traits aux membres de groupes subordonnés de sorte que les traits avantageux pour les membres des groupes dominants sont les plus prescrits. En outre, il apparaît que des variables dispositionnelles peuvent également influencer la propension à utiliser la prescription. En

\footnotetext{
${ }^{1}$ D'autres modèles ont été testés. L'impact de l'adhésion au sexisme bienveillant a ainsi été testé à différents niveaux. Ces analyses ont indiqué que le sexisme bienveillant n'avait d'effet direct ni sur la perception d'un bénéfice à prescrire des traits de sociabilité aux femmes, ni sur la prescription elle-même.
} 
effet, les hommes qui adhèrent à des idéologies sexistes, justifiant le statut subordonné des femmes, sont plus prompts à percevoir leur avantage dans certains des traits possédés par les femmes et par conséquent à leur prescrire ces traits que des hommes moins sexistes.

\section{DISCUSSION ET CONCLUSION}

Les études que nous avons présentées indiquent que les femmes, membres d'un groupe subordonné, se voient prescrire des traits et des émotions liés à la sociabilité. Or, ce type de traits correspond à des comportements de soumission et de respect. Quant aux hommes, membres d'un groupe dominant, ce sont des traits liés à la compétence et des émotions liées au pouvoir qui leur sont prescrits. Les données obtenues par Hess et al. (2005) prouvent que le type d'émotions prescrites dépend du statut du groupe (dominant vs. subordonné) auquel appartient l'individu. En outre, les traits prescrits aux subordonnés (généralement de la sociabilité) sont des traits susceptibles de les maintenir dans leur statut subordonné tout en assurant leur coopération avec les membres du groupe dominant. Les études que nous avons présentées montrent également que les femmes qui ne se conforment pas aux prescriptions de genre sont sanctionnées. Bien qu'il existe moins d'études à ce sujet, il semble que les hommes eux aussi puissent être victimes de sanctions lorsqu'ils ne se conforment pas aux prescriptions de genre. Les études présentées dans cet article indiquent également que la tendance prescriptive des individus prédit leur propension à sanctionner les déviants. Enfin, il apparaît que l'utilité des traits pour le groupe dominant détermine la mesure dans laquelle ils sont prescrits. Il semble donc que plus un trait est utile aux dominants, plus il est prescrit et que les individus qui ne se conforment pas à ces prescriptions sont sanctionnés d'une manière ou d'une autre. En d'autres termes, les résultats des études sur la composante prescriptive des stéréotypes sont en ligne avec l'idée selon laquelle les prescriptions stéréotypiques permettraient aux membres du groupe dominant de maintenir les subordonnés dans une position qui leur est avantageuse.

En ce qui concerne le lien entre prescriptions stéréotypiques et maintien du système social, Jost et Kay (2005) ont montré que le simple fait de rappeler les stéréotypes complémentaires de genre menait à accepter davantage le système social en place. Ainsi, lorsque les stéréotypes qui 
présentent l'homme et la femme comme complémentaires (l'un étant compétent, assertif et ambitieux ; l'autre étant sociable, douce et sensible) sont rendus plus accessibles, la tendance qu'ont les individus à percevoir le système social existant comme légitime est plus importante. En outre, il a été montré que les stéréotypes qui prescrivent de la sociabilité (et proscrivent la compétence) aux membres de certains groupes, en particulier aux femmes, contribuent au maintien de ces derniers dans un statut subordonné (voir les recherches de Dardenne et al., 2007 ; Davies et al., 2005 ; Dickhäuser \& Stiensmeier-Pelster, 2002 ; Eccles, 2007 ; Spencer et al., 1999). Cependant, peu d'études se sont penchées sur le lien entre prescriptions stéréotypiques et maintien du statu quo au sein d'un système social et, par conséquent, un certain nombre de questions sont encore en suspens. On peut notamment se demander si les prescriptions stéréotypiques contribuent réellement au maintien du statu quo dans une société donnée. En effet, si les stéréotypes prescriptifs ont bien pour fonction le maintien de la stabilité du système et de l'avantage des dominants, alors les systèmes les plus prescriptifs devraient être les plus stables et les dominants devraient y bénéficier de plus d'avantages que dans des systèmes moins prescriptifs. Cette hypothèse pourrait être envisagée dans des recherches appliquées, qui étudient des groupes sociaux réels. Ainsi, on peut s'attendre à ce que les individus expriment davantage de prescription dans les sociétés très stables que dans les sociétés en mutation ou qui connaissent des conflits sociaux. De même, lorsque les membres des groupes subordonnés s'auto-prescrivent des traits de sociabilité et des traits de compétence susceptibles de profiter aux dominants, on peut s'attendre à rencontrer peu de conflits sociaux. Dans ce sens, Jost et ses collègues (2003) ont déjà montré, dans une série d'études réalisées aux États-Unis, que les membres des groupes désavantagés adhéraient fortement aux croyances justifiant le système et soutenaient les idéologies favorisant les membres des groupes dominants. Cependant, il n'existe à notre connaissance aucune étude de terrain se focalisant sur les tendances prescriptives des subordonnés. De même, il n'existe pas non plus d'étude comparant les tendances prescriptives de groupes sociaux issus de systèmes sociaux différents. Ce type de recherches permettrait de vérifier si effectivement davantage de prescription mène à davantage de stabilité et cela permettrait en outre de valider notre hypothèse avec d'autres populations que les hommes et les femmes.

Jusqu'à présent, on ne sait pas dans quelle mesure il existerait, chez les membres de groupes dominants, une motivation consciente à maintenir le statu quo et à utiliser les stéréotypes à cet effet. Il serait par conséquent intéressant d'explorer l'aspect motivationnel de la prescription. Selon Jost 
et Banaji (1994), les stéréotypes prescriptifs seraient liés à des processus d'ordre motivationel. Selon ces auteurs, la composante descriptive des stéréotypes servirait plutôt une fonction cognitive (organiser et structurer les informations rencontrées au quotidien), tandis que la composante prescriptive des stéréotypes serait liée à des préoccupations d'ordre motivationnel et idéologique (voir aussi Jost \& Burgess, 2000 ; Jost et al., 2003). À notre connaissance, il n'existe cependant aucune recherche empirique qui teste directement ces hypothèses.

Si la fonction des stéréotypes prescriptifs est bien de maintenir le statu quo et l'avantage des dominants, alors il est probable qu'un ensemble de prescriptions pèse également sur les membres des groupes dominants. En effet, si ces derniers veulent conserver leur position dominante, il est nécessaire qu'ils fassent preuve d'un certain niveau de compétence et qu'ils ne montrent aucun signe de faiblesse ou de soumission. Les recherches existantes se centrent principalement sur les traits prescrits aux subordonnés, et très peu sur ce qui est prescrit aux dominants. Il existe quelques études qui explorent les caractéristiques prescrites aux hommes, membres d'un groupe dominant (voir par exemple Hess et al., 2005 ; Prentice \& Carranza, 2002 ; Sandnabba \& Ahlberg, 1999 ; Timmers et al., 2003). Mais aucune recherche, à notre connaissance, ne s'intéresse à l'utilité de ces traits pour le groupe dominant ou à l'impact de ces prescriptions sur la stabilité du système. Il serait par conséquent utile que la recherche future se penche davantage sur les prescriptions dont les groupes de haut statut sont la cible et sur leurs conséquences.

Des recherches que nous avons décrites, il apparaît également qu'il peut y avoir un impact de variables dispositionnelles sur les tendances prescriptives. Une de nos études (Delacollette et al., 2010) indique en effet un impact du niveau et du type de sexisme sur la perception du bénéfice que la sociabilité des femmes représente pour les hommes et, par conséquent, sur la prescription de ces traits aux femmes. D'autres variables dispositionnelles sont bien entendu susceptibles d'influencer les tendances prescriptives. Ainsi, il est probable que la tendance à la justification du système (Jost \& Banaji, 1994) et l'orientation vers la dominance sociale (SDO, Sidanius \& Pratto, 1999) influencent fortement la propension à prescrire aux subordonnés des traits avantageux pour les dominants. Ce lien entre variables dispositionnelles et stéréotypes prescriptifs pourrait faire l'objet d'une nouvelle lignée de recherche.

Enfin, il apparaît dans notre revue de la littérature que la recherche s'est principalement penchée sur les conséquences de la prescription au niveau intergroupe, mais très peu au niveau interindividuel. Or, il est probable que 
la prescription de caractéristiques au niveau groupal ait des conséquences au niveau des relations interpersonnelles. Par exemple, la prescription de sociabilité aux femmes et de compétence aux hommes en tant que groupes entraîne très probablement une série d'attentes au niveau des relations entre individus. Ces attentes pourraient entre autres se manifester dans la sphère privée, lors de la recherche d'un partenaire ou dans les attentes qu'un homme ou une femme a vis-à-vis de son conjoint. À nouveau, ces hypothèses ouvrent la voie à de nouvelles lignées de recherche, telles que des recherches sur le lien entre prescription et relation amoureuse ou encore sur le maintien des stéréotypes via les interactions interindividuelles.

Il semble, en conclusion, que la composante prescriptive des stéréotypes est un outil particulièrement subtil de contrôle social. Elle sert les avantages des groupes dominants en poussant les subordonnés à adopter des caractéristiques bénéfiques aux dominants. Elle permettrait en outre de maintenir les subordonnés dans leur position désavantageuse sans pour cela utiliser la coercition. En effet, de par le poids de la prescription et la menace de la sanction, les membres des groupes subordonnés finissent par se conformer d'eux-mêmes aux stéréotypes qui leur prescrivent une haute sociabilité mais peu de compétence. Par conséquent, ils sont moins susceptibles de rechercher un statut plus élevé et davantage de pouvoir. Néanmoins, afin de mieux comprendre ce processus, de nombreuses voies de recherche restent encore à explorer, telles que l'aspect motivationnel de la prescription, le lien entre prescription et stabilité du système, la prescription portant sur les membres des groupes dominants, l'impact des variables dispositionnelles sur la prescription, ou encore les effets de la prescription au niveau interindividuel.

\section{BIBLIOGRAPHIE}

Adams, G., Garcia, D. M., PurdieVaughns, V., \& Steele, C. M. (2006). The detrimental effects of a suggestion of sexism in an instruction situation. Journal of Experimental Social Psychology, 42, 602-615.

Adorno, T. W., Frenkel-Brunswik, E., Levinson, D. J., \& Sanford, R. N. (1950). The authoritarian personality. New York: Harper \& Row.

Alexander, M. G., Brewer, M. B., \& Herrmann, R. K. (1999). Images and affect: A functional analysis of out-group stereotypes. Journal of Personality and Social Psychology, 77, 78-93.

Asch, S. E. (1946). Forming impressions of personality. Journal of Abnormal and Social Psychology, 41, 258-290.

Benokraitis, N. V. (1997). Subtle sexism. Current Practices and prospects for change. Thousand Oaks: Sage Publications.

Burgess, D., \& Borgida, E. (1999). Who women are, who women should 
be: Descriptive and prescriptive gender stereotyping in sex discrimination. Psychology, Public Policy and Law, 5, 665-692.

Cialdini, R. B., \& Trost, M. R. (1998). Social influence: Social norms, conformity and compliance. In Gilbert, D. T., Fiske, S. T., \& Lindzey, G. (Eds), The handbook of social psychology, Vol. 2 (4th ed.). New York: McGraw-Hill.

Conway, M., Pizzamiglio, M. T., \& Mount, L. (1996). Status, communality, and agency: Implications for stereotypes of gender and other groups. Journal of Personality and Social Psychology, 71, 25-38.

Dardenne, B., Delacollette, N., Grégoire, C., \& Lecocq, D. (2006). Latent structure of the french validation of the Ambivalent Sexism Inventory: l'Echelle de Sexisme Ambivalent. L'Année psychologique, 106, 235-264.

Dardenne, B., Dumont, M., \& Bollier, T. (2007). Insidious dangers of benevolent sexism: Consequences for Women's performance. Journal of Personality and Social Psychology, 93, 764-779.

Delacollette, N., Dumont, M., \& Dardenne, B (2010). Tell me what is beneficial to dominants and I'll tell you what is prescribed to subordinates. Prescriptive stereotypes in intergroup settings. Manuscript in prep.

Delacollette, N., Dardenne, B., \& Dumont, M. (2010). Benevolent sexism, men's advantages and prescription of warmth to women. Manuscript in prep.

Dickhäuser, O., \& Stiensmeier-Pelster, J. (2002). Gender differences in computer work: Evidence for the model of achievement-related choices. Contemporary Educational Psychology, 27, 486-496.

Diekman, A. B., \& Goodfriend, W. (2006). Rolling with the changes: a role congruity perspective on gender norms. Psychology of Women Quarterly, 30, 369-383.
Eagly, A. H., \& Karau, S. T. (2002). Role congruity theory of prejudice toward female leaders. Psychological Review, 109, 573-598.

Eagly, A. H., Makhijani, M. G., \& Klonsky, B. G. (1992). Gender and the evaluation of leaders: A meta-analysis. Psychological Bulletin, 111, 3-22.

Eccles, J. S. (2007). Where are all the women? Gender differences in participation in physical science and engineering. In W. M. Williams \& S. J. Ceci (Eds.) Why aren't women in science: Top researchers debate the evidence. Washington, DC, US: American Psychological Association.

Fiske, S. T., Bersoff, D. N., Borgida, E., Deaux, K., \& Heilman, M. E. (1991). Social science research on trial : Use of sex stereotyping research in Price Waterhouse vs. Hopkins. American Psychologist, 46, 1049-1060.

Fiske, S. T., Cuddy, A. J., Glick, P., \& Xu, J. (2002). A model of (often mixed) stereotype content: Competence and warmth respectively follow from perceived status and competition. Journal of Personality and Social Psychology, 82, 878-902.

Fiske, S. T., Xu, J., Cuddy, A. C., \& Glick, P. (1999). (Dis)respecting versus (dis)liking : Status and interdependence predict ambivalent stereotypes of competence and warmth. Journal of Social Issues, $55,473-489$.

Gill, M. J. (2004). When information does not deter stereotyping: Prescriptive stereotyping can foster bias under conditions that deter descriptive stereotyping. Journal of Experimental Social Psychology, 40, 619-632.

Glick, P., \& Fiske, S. T. (1996). The Ambivalent Sexism Inventory: Differentiating hostile and benevolent sexism. Journal of Personality and Social Psychology, 70, 491-512.

Glick, P., \& Fiske, S. T. (1999). Sexism and other « isms »: Interdependence, status, and the ambivalent content of stereotypes. 
In Swann, Jr., W. B., Gilbert, L. A., \& Langlois, J. (Eds.), Sexism and Stereotypes in Modern Society: The Gender Science of Janet Taylor Spence. Washington: American Psychological Association.

Glick, P., \& Fiske, S. T. (2001). Ambivalent stereotypes as legitimizing ideologies: differentiating paternalistic and envious prejudice. In Jost, J. T. \& Major, B. (Eds.) The Psychology of Legitimacy: Emerging Perspectives on Ideology, Justice, and Intergroup Relations. Cambridge: Cambridge University Press.

Hamilton, D. L., \& Stroessner, S. J. (1994). Social cognition and the study of stereotyping. In Devine, P. G., Hamilton, D. L., \& Ostrom, T. M. (Eds), Social cognition: impact on social psychology (pp. 291-321). San Diego: Academic Press.

Heilman, M. E. (2001). Description and prescription: How gender stereotypes prevent women's ascent up the organizational ladder. Journal of Social Issues, 57, 657-674.

Heilman, M. E., \& Chen, J. J. (2005). Same behaviour, different consequences: reactions to men's and women's altruistic citizenship behaviour. Journal of Applied Psychology, 90, 431-441.

Heilman, M. E., \& Okimoto, T. G. (2007). Why are women penalized for success at male tasks?: The implied communality deficit. Journal of Applied Psychology, 92, 81-92.

Heilman, M. E., Wallen, A. S., Fuchs, D., \& Tamkins, M. M. (2004). Penalties for success: Reactions to women who succeed at male gender-typed tasks. Journal of Applied Psychology, 89, 416-427.

Herbert, J., \& Stipeck, D. (2005). The emergence of gender differences in children's perceptions of their academic competence. Journal of Applied Developmental Psychology, 26, 276-295.

Hess, U., Adams, R. B., \& Kleck, R. E. (2005). Who may frown and who should smile? Dominance, affiliation, and the display of happiness and anger. Cognition and Emotion, 19, 515-536.

Jackman, M. (1994). The Velvet Glove: Paternalism and conflict in gender, class and race relations. Berkeley: University of California Press.

Jost, J. T., \& Banaji, M. R. (1994). The role of stereotyping in system-justification and the production of false-consciousness. British Journal of Social Psychology, 33, 1-27. Jost, J. T., \& Burgess, D. (2000). Attitudinal ambivalence and the conflict between group and system justification motives in low status groups. Personality and Social Psychology Bulletin, 26, 293-305.

Jost, J. T., Burgess, D., \& Mosso, C. O. (2001). Conflicts of legitimation among self, group, and system. The integrative potential of system justification theory. In Jost, J. T. and Major, B. (Eds), The Psychology of Legitimacy: Emerging perspectives on ideology, justice, and intergroup relations. Cambridge: Cambridge University Press.

Jost, J. T., \& Hunyady, O. (2002). The psychology of system justification and the palliative function of ideology. European Review of Social Psychology, 13, 111-153.

Jost, J. T., \& Kay, A. C. (2005). Exposure to benevolent sexism and complementary gender stereotypes: Consequences for specific and diffuse forms of system justification. Journal of Personality and Social Psychology, 88, 498-509.

Jost, J. T., Pelham, B. W., \& Carvallo, M. R. (2002). Non-conscious forms of system justification: Implicit and behavioral preferences for higher status groups. Journal of Experimental Social Psychology, 38, 586-602.

Jost, J. T., Pelham, B. W., Sheldon, O., \& Sullivan, B. N. (2003). Social inequality and the reduction of ideological dissonance on behalf of the system: evidence of enhanced system justification among the disadvantaged. European Journal of Social Psychology, 33, 13-36. 
Judd, C. M., James-Hawkins, L., Yzerbyt, V., \& Kashima, Y. (2005). Fundamental dimensions of social judgment: Understanding the relations between judgments of competence and warmth. Journal of personality and Social Psychology, 89, 899-913.

Jussim, L., Coleman, L. M., \& Lerch, L. (1987). The nature of stereotypes: A comparison and integration of three theories. Journal of Personality and Social Psychology, 52, 536-546.

Katz, D., \& Braly, K. (1933). Racial stereotypes in one hundred college students. Journal of Abnormal and Social Psychology, 28, 280-290.

Leyens, J.-P., Yzerbyt, V., \& Schadron, G. (1994). Stereotypes and social cognition. London: Sage Publications.

Manstead, A. S. R., Hewstone, M., Fiske, S. T., Hogg, M. A., Reis, H. T., Semin, G. R. (Eds) (1995). The Blackwell encyclopedia of social psychology. Cambridge: Blackwell.

Moya, M., Glick, P., Exposito, F., de Lemus, S., \& Hart, J. (2007). It's for your own good : Benevolent sexism and women's reactions to protectively justified restrictions. Personality and Social Psychology Bulletin, 33, 1421-1434.

Phalet, K., \& Poppe, E. (1997). Competence and morality dimensions of national and ethnic stereotypes: A study in six Eastern-European countries. European Journal of Social Psychology, 27, 703-723.

Poppe, E., \& Linssen, H. (1999). In-group favouritism and the reflection of realistic dimensions of difference between national states in Central and Eastern European nationality stereotypes. British Journal of Social Psychology, 38, 85-102.

Prentice, D. A., \& Carranza, E. (2002). What women and men should be, shouldn't be, are allowed to be, and don't have to be: The contents of prescriptive gender stereotypes. Psychology of Women Quarterly, 26, 269-281.
Rosenberg, S., Nelson, C., \& Vivekananthan, P. S. (1968). A multidimensional approach to the structure of personality impressions. Journal of Personality and Social Psychology, 9, 283-294.

Rubin, Z., \& Peplau, L. A. (1975). Who believes in a just world? Journal of Social Issues, 31, 65-90.

Rudman, L. A., \& Fairchild, K. (2004). Reactions to counterstereotypic behaviour: The role of backlash in cultural stereotype maintenance. Journal of Personality and Social Psychology, 87, 157-176.

Rudman, L. A., \& Glick, P. (1999). Feminized management and backlash toward agentic women: The hidden costs to women of of a kinder, gentler image of middle managers. Journal of Personality and Social Psychology, 77, 1004-1010.

Rudman, L. A., \& Glick, P. (2001). Prescriptive gender stereotypes and backlash toward agentic women. Journal of Social Issues, 57, 743-762.

Sagar, H. A., \& Schofield, J. W. (1980). Racial and behavioral cues in Black and White children's perceptions of ambiguously aggressive acts. Journal of Personality and Social Psychology, 39, 590-598.

Sandnabba, N. K., \& Ahlberg, C. (1999). Parents' attitudes and expectations about children's cross-gender behavior. Sex Roles, 40, 249-263.

Schneider, D. J. (2004). The psychology of stereotyping. New York: Guilford Press.

Sidanius, J., \& Pratto, F. (1999). Social dominance: An intergroup theory of social hierarchy and oppression. New York: Cambridge University Press.

Spence, J. T., \& Helmreich, R. L. (1978). Masculinity and feminity: Their psychological dimensions, correlates, and antecedents. Austin: University of Texas Press.

Spencer, S. J., Steele, C. M., \& Quinn, D. M. (1999). Stereotype threat and women's math performance. Journal of Experimental Social Psychology, 35, 4-28. 
Spears, R., Oakes, P. J., Ellemers, N., \& Haslam, S.A. (1997). The social psychology of stereotyping and group life. Malden: Blackwell.

Stoppard, J. M., \& Gunn Gruchy, C. D. (1993). Gender, context, and expression of positive emotion. Personality and Social Psychology Bulletin, 19, 143-150.

Tafarodi, R. W., \& Swann, W., B., Jr. (1995). Self-liking and self-competence as dimensions of global self-esteem: Initial validation of a measure. Journal of Personality Assessment, 65, 322-342.

Tajfel, H. (1981). Social stereotypes and social groups. In Turner, J. C. \& Giles, H. (Eds), Intergroup Behaviour. Oxford: Basil Blackwell.
Timmers, M., Fischer, A. H., \& Manstead, A. S. R. (2003). Ability versus vulnerability: Beliefs about men's and women's emotional behaviour. Cognition and Emotion, 17, 41-63.

Yzerbyt, V., Provost, V., \& Corneille, O. (2005). Not competent but warm... Really? Compensatory stereotypes in the french-speaking world. Group Processes and Intergroup Relations, 8, 291-308.

Yzerbyt, V., \& Schadron, G. (1994). Stéréotypes et jugement social. In Bourhis, R. Y. \& Leyens, J.-P. (Eds). Stéréotypes, discrimination et relations intergroupes. Liège : Mardaga.

Wyer, R. S. Jr. (Ed) (1998). Stereotype activation and inhibition. Mahwah: Lawrence Erlbaum Associates Publishers. 